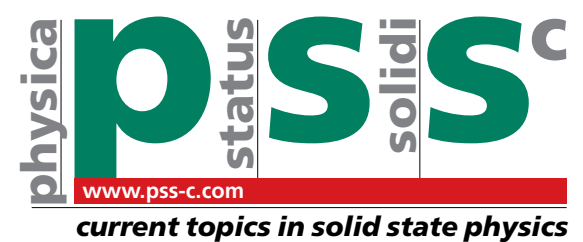

\title{
Resonant nonlinear studies of trapped OD-microcavity polaritons
}

\section{Gaël Nardin ${ }^{\star}$, Ounsi El Daïf, Taofiq K. Paraïso, Augustin Baas, Maxime Richard, Francois Morier-Genoud, and Benoit Deveaud}

Institut de Photonique et d'Électronique Quantiques, École Polytechnique Fédérale de Lausanne (EPFL), Station 3, 1015 Lausanne, Switzerland

Received 10 September 2007, accepted 3 December 2007

Published online 18 February 2008

PACS 71.20.Nr, 71.36.+c

* Corresponding author: e-mail gael.nardin@epfl.ch, Phone: +41-21-693 54 49, Fax: +41-21-693 4525

We performed studies on microcavity polaritons trapped along the three dimensions of space, under resonant excitation on a confined lower polariton state. We observed various nonlinear behaviors as a function of the pump power, without any apparent loss of the strong-coupling.
That may be understood as effects of Coulomb interaction. Indications of bistable behaviors in the system are observed and discussed.
1 Introduction Most breakthroughs in semiconductor physics and technology over the last thirty years originated from quantum confinement of elementary excitations along one, two, or three spatial dimensions [1] and from the improvement of their coupling to the electromagnetic field. Indeed, confinement in semiconductor structures allows design and shaping of their electrical and optical properties, and the study of various effects, ranging from the Purcell effect [2] to full quantum confinement. Such confinement allows to control the emission properties of matter, and can be used for applications in many fields, ranging from optoelectronics to quantum information.

Microcavity polaritons are 2D quasi-particles arising from the strong-coupling between a quantum well exciton and a cavity photon. Thanks to their excitonic content, polaritons can interact strongly, added to their peculiar dispersion shape, this allowed to observe polariton parametric scattering where two polaritons in an initial pump state $\left(E_{p}, \mathbf{k}_{\mathbf{p}}\right)$, scatter towards idler $\left(E_{i}, \mathbf{k}_{\mathbf{i}}\right)$ and signal $\left(E_{s}, \mathbf{k}_{\mathbf{s}}\right)$ states, along the lower polariton (LP) dispersion curve [3, 4]. This process is similar to the optical parametric oscillator (OPO). It has lead to the observation of several phenomena of interest as giant parametric amplification [5] or coherent control of polaritons [6] along with a varied phenomenology. For one given pump state, the allowed couples of signals and idlers are described by 8 -shaped lines in the 2D reciprocal space, as was observed by [7]. One interesting pump state is at the so-called magic angle, for which the signal state corresponds to the bottom of the dispersion, that is the lower polariton ground state $\left(E_{0}, \mathbf{k}=\mathbf{0}\right)$. This situation shows the smallest density threshold for parametric conversion $[3,4,8]$.

\section{Experimental facts}

2.1 Sample We fabricated a GaAs/AlAs $2 \mathrm{D}$ semiconductor $\lambda$-microcavity with an embedded InGaAs quantum well, giving rise to a Rabi splitting of $3.5 \mathrm{meV}$. We have designed and realized $6 \mathrm{~nm}$ high mesas of $9 \mu \mathrm{m}$ in diameter on the top of the spacer layer, which act as OD traps for the photon mode. We previously demonstrated and characterized strong-coupling in 0 and 2 dimensions for the various mesa sizes [9]. The 0D structure shows a very high-Q factor of $\sim 21000$, thanks to the fact that the confining mesas have sizes smaller than the typical optical disorder in GaAs microcavities [10,11].

In such a confined structure, the dispersion curve shows discrete levels, delocalised in the reciprocal (k-)space, and thus with an angular broadening, as we have previously observed $[12,13]$. This should favor parametric processes, due to the relaxation of the wave vector conservation condition. 
In the scope of the quest for parametric amplification in our system, we present here studies under resonant excitation on a $0 D$ trapped polariton state in a $9 \mu$-mesa. We performed detailed studies of the behavior of the photoluminescence (PL) emission of the ground state of the system, while increasing the density of created population, which is linearly linked to the pump power.

While the non-resonant excitation at high energy of the system creates free carriers contributing to the total density of population and thus to the loss of the strong-coupling regime [12-14], resonant excitation of the system creates only polaritons. In order to excite selectively a given eigenstate, one needs to be able to observe these states. As they are defined in the wave vectors' basis, it is necessary to observe the reciprocal space (accessible through angle resolved imaging and excitation) of the sample in order to be able to select which state to populate.

2.2 Experimental set-up Excitation of the sample is performed with a continuous tunable TiSa laser, the excitation angle is $\simeq 10^{\circ}$, and the energy of the laser is 1486.3 $\mathrm{meV}$. By imaging the $\mathrm{k}$-space of the sample on the slit of a spectrometer, we obtain angle resolved spectra, and thus image the k-space of the system. Fig. 1(a) shows the PL spectrum obtained in this configuration for a $9 \mu \mathrm{m}$ diameter mesa, at zero detuning for the lower confined polariton mode, while exciting non-resonantly (at high energy) the system. One can see that the confined states have lobes depending on the angle of detection. (These lobes correspond to the quantum number $m[13,15])$ We therefore chose the laser pumping angle so that it corresponds to a lobe on the image shown on Fig. 1(a), in order to, as much as possible, improve its coupling to the system. The energy chosen shows an available signal-idler couple of states fulfilling the energy conservation with a precision better than the linewidth: $E_{\text {idler }}-E_{\text {pump }}=0.87 \mathrm{meV}$ and $E_{\text {pump }}-E_{\text {signal }}=0.90 \mathrm{meV}$, where the signal state is the LP ground confined state. These values are smaller than the linewidth of the confined photon: $0.90-0.87=0.03 \mathrm{meV}$ $<0.1 \mathrm{meV} \simeq \gamma_{0 D}$, and thus of the confined polariton.

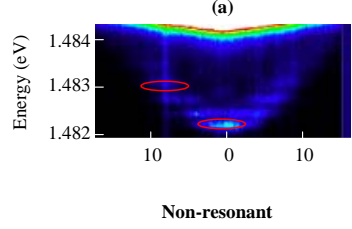

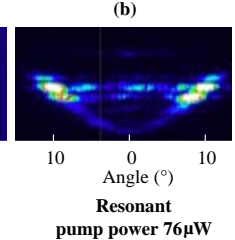

(c)

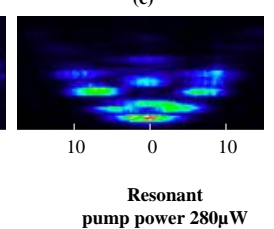

Figure 1 (a) Dispersion in photoluminescence under nonresonant excitation of the $9 \mu \mathrm{m}$-diameter mesa lower polariton at zero detuning, the white signal at high energy is the $2 \mathrm{D}$ lower polariton (at the exciton's energy). The pump state chosen and its potential signal below are circled in red. (b) Resonant pumping. (c) Emission at the nonlinear threshold. Mentioned pump powers correspond to the experiment shown on Fig. 2.
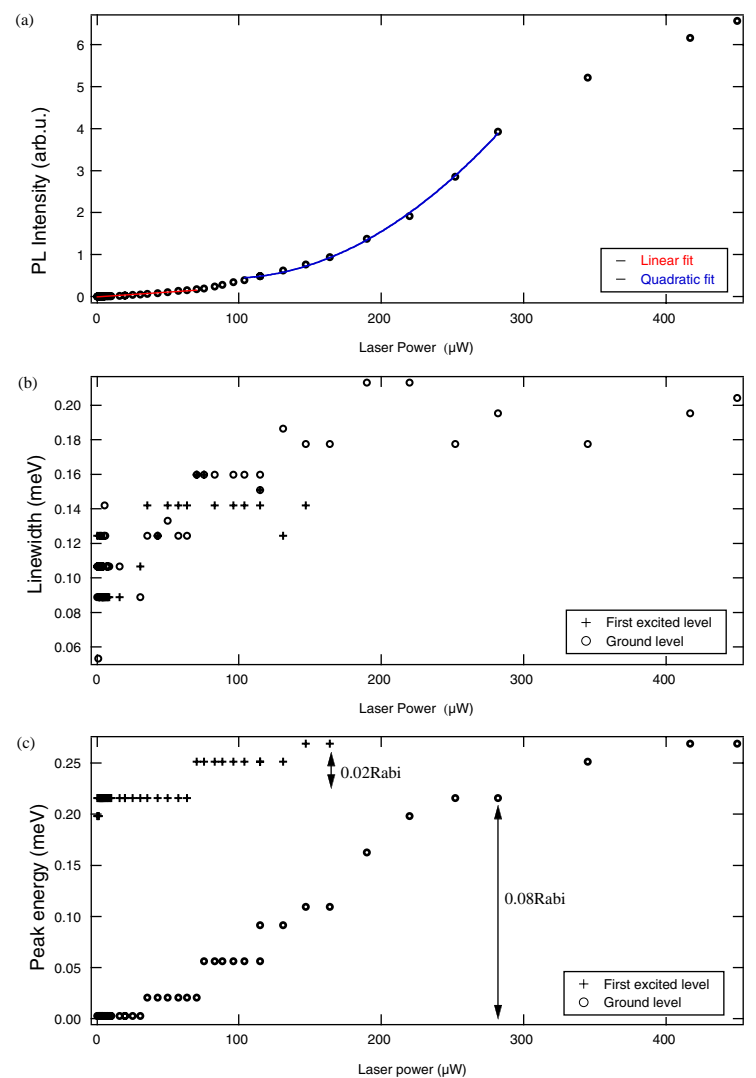

Figure 2 (a) PL intensity of the lower polariton ground confined level as a function of the laser power (color online). (b) Linewidth of the lower polariton ground confined level (circles), and first excited level (crosses). (c) Energy shift of the lower polariton ground confined level (circles) and first excited level (crosses), the ratio of the largest shift over the Rabi splitting is given.

3 Results Results obtained are shown on Fig. 2, three regimes are distinguishable for the PL intensity evolution: linear, quadratic and then a saturation. We integrated the whole angular extension of the OD ground state. The signal shown on Fig. 2(a) is thus directly proportional to the whole population of this state. A quadratic fit describes well the evolution between $60 \mu \mathrm{W}$ and $260 \mu \mathrm{W}$. We were easily able to trace the evolution of the linewidth and energy of this state on Figs. 2(b) and (c). There is indeed a small increase of the linewidth due to density effects. There is also a slight blueshift of the resonance energy. This blueshift being small compared to the Rabi splitting ( 0.08 times the Rabi), we can fairly assume that the system stays in strong coupling regime during the first two regimes. We discuss below the high excitation power case.

4 Signs of bistability? The first linear regime is attributed to linear relaxation channels, while the quadratic regime shows the contribution, as in the 2D case, of polariton-polariton scattering, namely, parametric luminescence [16]. We did not observe any luminescence at the 
idler state, but its intensity is expected to be extremely low $[4,5]$ : it shows a very small photonic component (due to its positive detuning) and it has many relaxation and coupling channels possible with lower energy states or with the $2 \mathrm{D}$ exciton. The following saturation is attributed to the fact that the polariton mode blueshifts while the population increases, leading to a detuning with respect to the excitation.

Two phenomena can be considered for the blueshift of the polariton mode: the polariton-polariton interaction [8, 16] and the bleaching of the strong coupling regime [17]. It has been shown for the first phenomenon by $[18,19]$ and for the second by [20] that both phenomena can lead to optical bistability in the case of 2D polaritons. Indeed, we were able to fit the peculiar shapes of the ground state intensity evolution by a cubic polynomial expression, characteristic of bistability, by inverting the two axes of the plotted graphs. Fig. 3(a) shows again the evolution of the ground mode luminescence intensity under increasing excitation power, more points have been taken at critical positions such as apparent regime change. Fig. 3(b) shows the same data as Fig. 3(a), but the pump power is plotted as a function of the emitted PL intensity. A cubic polynomial fits very well the measurements. In the following, we try to determine which source of blueshift is dominating in our system, and if it can leads to bistability.
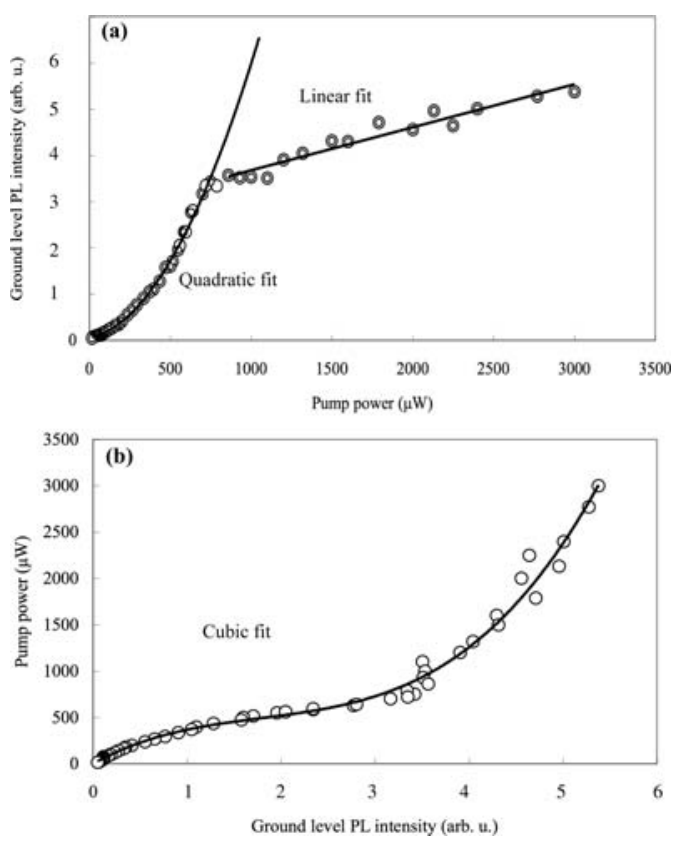

Figure 3 Evolution of the ground state PL intensity of a $9 \mu \mathrm{m}$ mesa, seen in two different ways: on one graph the PL intensity is shown as a function of the pump power, in the other graph it is the pump power which is seen as a function of the PL intensity.

4.1 Degenerate scattering Reference [19] shows that bistability can be obtained through degenerate para- metric oscillations, and reference [18] provides an experimental evidence in the case of normal incidence excitation. Polaritons scatter coherently within the pumped state (where energy and phase-matching conditions are obviously fulfilled) yielding a nonlinear dependence of the polariton field with the pump power. Polaritons then relax linearly through phonons towards the ground state. The evolution of the ground state population is thus representative of the evolution of the pumped state population. In this case, the system shows a bistable behaviour for a positive detuning of the laser with respect to the pumped polariton energy. The results shown on Fig. 3 were performed with such a positive detuning, but didn't show any hysteresis or instability behavior. For various detunings, the evolution of the PL intensity was never sharper than what is shown here. We therefore ruled out the degenerate scattering assumption.

\subsection{Bleaching of the strong-coupling regime}

Reference [20] suggests another phenomena yielding a bistable behavior in the dependence of a polariton population with respect to a pump intensity: the bleaching of the strong coupling regime, while exciting resonantly a polariton state. The developed model uses the experimental parameters of [21] (an AlGaAs/AlAs cavity with five embedded GaAs quantum wells). According to Ref. [20], bistability depends on a broadening factor directly related to the linewidth of the exciton resonance. If the latter is too large, no bistability is observed, but simply a cubic behavior. We could not tune this parameter, as we worked on one sample showing a given linewidth, and could therefore not conclude on this point.

Future samples with various exciton linewidths may allow us to observe bistable behaviour and confirm the assumption of the bleaching of the strong coupling.

5 Balance and conclusions We performed various studies looking for a massive redistribution of the polariton population towards a single state, this would open the way to parametric oscillation and amplification. We observed quadratic regimes, indicating that polaritonpolariton scattering takes place in the relaxation processes towards the ground confined polariton states, but did not observe any oscillation, most probably because the nonlinear thresholds were too high, compared to the strongto-weak coupling cross-over threshold. A solution to overcome this effect would be to increase the number of quantum wells within the cavity. This would yield an increase of the strong-to-weak-coupling transition without affecting the nonlinear thresholds.

We have observed cubic dependances, indicating that bistability, as described by [20], would be possible in our mesa structures. In this case, the subsequent quantization of the modes would allow to observe efficient fast optical switching in future samples.

Parametric effects in between confined states and between confined and extended states can also be expected, 
and may allow the observation of coherence transfer between states or coherent effects [22]. The conception of new trap shapes, in particular coupled traps, would allow the possibility of seeing such effects between polaritons trapped in different mesas.

As one can see, the fabrication of new sample structures (in terms of composition or of trap shapes) will allow a wide range of phenomena to be observed, ranging from basic effects to applications.

Acknowledgements We thank Stefano Portolan and Davide Sarchi for rich discussions, and Jean-Philippe Brantut for experimental support. We acknowledge financial support from the Swiss National Centre for Competence in Research (NCCR) Quantum Photonics.

\section{References}

[1] D. Bimberg, M. Grundmann, and N. N. Ledentsov, Quantum Dot Heterostructures (Wiley, 1999).

[2] E. M. Purcell, Phys. Rev. 69, 681 (1946).

[3] P. G. Savvidis, J. J. Baumberg, R. M. Stevenson, M. S. Skolnick, D. M. Whittaker, and J.S. Roberts, Phys. Rev. Lett. 84(7), 1547-1550 (2000).

[4] R. M. Stevenson, V. N. Astratov, M. S. Skolnick, D. M. Whittaker, M. Emam-Ismail, A. I. Tartakovskii, P. G. Savvidis, J. J. Baumberg, and J. S. Roberts, Phys. Rev. Lett. 85(17), 3680-3683 (2000).

[5] M. Saba, C. Ciuti, J. Bloch, V. Thierry-Mieg, R. Andr, L. S. Dang, S. Kundermann, A. Mura, G. Bongiovanni, J. L. Staehli, and B. Deveaud, Nature 414(Dec), 731 (2001).

[6] S. Kundermann, M. Saba, C. Ciuti, T. Guillet, U. Oesterle, J.L. Staehli, and B. Deveaud, Phys. Rev. Lett. 91(10), 107402 (2003).

[7] W. Langbein, Phys. Rev. B 70(20), 205301 (2004).

[8] C. Ciuti, P. Schwendimann, B. Deveaud, and A. Quattropani, Phys. Rev. B 62(8), R4825-R4828 (2000).

[9] O. E. Daïf, A. Baas, T. Guillet, J. P. Brantut, R. I. Kaitouni, J.L. Staehli, F. Morier-Genoud, and B. Deveaud, Appl. Phys. Lett. 88(6), 061105 (2006).

[10] D. Sanvitto, A. Daraei, A. Tahraoui, M. Hopkinson, P. W. Fry, D. M. Whittaker, and M. S. Skolnick, Appl. Phys. Lett. 86(19), 191109 (2005).

[11] O.E. Daï, OD Microcavity Polaritons. Trapping Lightmatter Quasiparticles (Ph. D. Thesis, EPFL, Lausanne, 2007).

[12] A. Baas, O.E. Daïf, M. Richard, J. P. Brantut, G. Nardin, R. I. Kaitouni, T. Guillet, V. Savona, J. L. Staehli, F. Morier-Genoud, and B. Deveaud, phys. stat. sol. (b) 243(10), 2311-2316 (2006).

[13] R. I. Kaitouni, O. E. Daï, A. Baas, M. Richard, T. Paraiso, P. Lugan, T. Guillet, F. Morier-Genoud, J. D. Ganire, J. L. Staehli, V. Savona, and B. Deveaud, Phys. Rev. B 74(15), 155311 (2006).

[14] O. E. Daïf, G. Nardin, T. K. Paraïso, A. Baas, M. Richard, F. Morier-Genoud, and B. Deveaud, submitted to Appl. Phys. Lett.

[15] P. Lugan, D. Sarchi, and V. Savona, phys. stat. sol. (c) 3(7), 2428-2431 (2006).
[16] C. Ciuti, P. Schwendimann, and A. Quattropani, Semicond. Sci. Technol. 18(10), S279-S293 (2003).

[17] R. Butté, G. Delalleau, A. I. Tartakovskii, M. S. Skolnick, V. N. Astratov, J. J. Baumberg, G. Malpuech, A. Di Carlo, A. V. Kavokin, and J.S. Roberts, Phys. Rev. B 65(20), 205310 (2002).

[18] A. Baas, J. P. Karr, H. Eleuch, and E. Giacobino, Phys. Rev. A 69(2), 023809 (2004).

[19] M. Wouters and I. Carusotto, Phys. Rev. B 75(7), 075332 (2007).

[20] A. Tredicucci, Y. Chen, V. Pellegrini, M. Börger, and F. Bassani, Phys. Rev. A 54(4), 3493-3498 (1996).

[21] T. B. Norris, J. K. Rhee, D. S. Citrin, M. Nishioka, and Y. Arakawa, Nuovo Cimento D 17(11-12), 1295-1303 (1995).

[22] S. Kundermann, T. Guillet, M. Saba, O.E. Daif, J.L. Staehli, and B. Deveaud, phys. stat. sol. (a) 201(3), 381388 (2004). 